\title{
Feature Exploring Cells
}

\section{Review of: The World of the Cell, 5th ed., by W.M. Becker, L.J. Kleinsmith, and J. Hardin; 2003; 802 pp.; Benjamin Cummings (San Francisco); ISBN: 0-8053-4852-2}

\author{
Reviewed by Mary J. Stewart, Department of Biological Sciences, \\ North Dakota State University
}

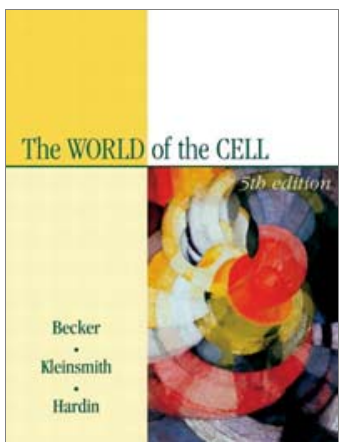

When I preview textbooks for my undergraduate cell biology course, I look for up-to-date content and an organization that allows flexibility in choosing the depth in which to discuss topics. It is impossible to learn or teach all of cell biology in depth in a one-semester course. Therefore, I look for a book that provides the foundations as well as more in-depth information but is organized in such a way that it is possible to make reading assignments that cover the "basics" or that also will go into more depth. I also try to look at the book from the perspective of a student who might be reading about a topic for the first time or perhaps reviewing and building on a topic previously studied in general biology. I ask whether the text is readable, the figures clearly illustrate concepts, and key ideas are emphasized or obscured by details. Several up-to-date cell biology textbooks are available, but I have adopted The World of the Cell (TWC) by Becker, Kleinsmith, and Harding because, in addition to being current, it also is very readable and versatile. After using two editions (initially the fourth and now the fifth) of TWC over the past 5 years, I still like it, and students have told me that it is well written and readable.

DOI: 10.1187/cbe.04-07-0051

E-mail address: Mary.Stewart@ndsu.nodak.edu.

TWC is versatile because of the way material is allocated between chapters, as well as the way that most chapters are organized. TWC is arranged into a logical series of topics that begins with the basic foundations of cell biology and continues with topics that span the breadth of cellular and molecular biology. Each chapter in TWC begins with a oneto four-page overview of the importance of the topic and the basic concepts of the topic before going into more detail in the rest of the chapter. Each chapter ends with a "perspective" section that summarizes key points. I find that it is often useful to read the perspectives before reading the chapter, and I suggest that students do the same. The chapters of TWC are organized into distinct sections that are delineated by bold and colored headings. Without detracting from readability, this feature allows one to choose whether or not to cover particular topics and the depth of coverage. For example, I routinely subdivide chapter 17 ("The Cell Cycle: DNA Replication, Mitosis, and Cancer"). I think of this chapter as having the three separate but related topics of 1) DNA replication and repair, 2) nuclear and cell division, and 3) growth control and cancer. Depending on the emphasis of the course and on time constraints, it is possible to pick and choose particular chapters to use or to omit. However, other chapters go hand-in-hand, one building on the concepts of the other. For example, it would be difficult to omit chapter 13, which discusses glycolysis and fermentation, and still include chapter 14, which deals with aerobic respiration.

The authors do a good job of illustrating the principles of a wide range of topics without obscuring the message with excessive details. This approach inevitably leads to the omission of details or information that an individual instructor might want to include. As an example, I'll use the topic of cell signaling in chapter 10. Chapter 10 describes signaling from G-protein coupled receptors, tyrosine kinase receptors, and receptor serine/threonine kinases, as well as apoptosis. The initial and heaviest emphasis is placed on signaling through G-protein coupled receptors and the subsequent production of second messengers. The epidermal growth factor receptor is then used to illustrate the basic structure and activation of tyrosine kinase receptors. The book nicely illustrates (in figure 10-18) how activation of tyrosine kinase receptors can lead to second messenger production and to Ras activation. The book clearly makes the point that Ras is active when bound to GTP and explains 
the role of guanine-nucleotide exchange factors and GTPase activating proteins in regulating whether Ras is in the GTPor GDP-bound state. However, the role of Ras in activating signaling through MAP kinases is mentioned only briefly and figure $10-18$ is very general with regard to MAP kinase signaling. In some respects, it would be useful to have more detail, such as information regarding the amplification of signals, the sequential activation of protein kinase cascades, or a more thorough explanation of pathways involving MAP kinases. However, if more comprehensive details on this or other topics were included, the strength of the book in providing breadth without excessive detail would be lost.

Interspersed throughout the book are descriptions of methods used in cell and molecular biology. Some methods are "classics" such as the use of centrifugation to separate cell components, appropriately placed in the context of intracellular compartments in chapter 12 . Some notable updates in chapter 16 include revision of figure 16-13, which illustrates restriction mapping, and figure 16-14, which shows automated DNA sequencing with the use of fluorescently labeled dideoxynucleotides. Chapter 21 includes nuclear run-on assays and a new section on microarrays as examples of methods to examine differential gene transcription. Both methods are clearly illustrated by figures that complement the text descriptions.

Several updates have been made to the fifth edition of TWC that reflect new advances in cell biology. For example, apoptosis, which was described in one page in the fourth edition, is now described in three and one-half pages with two new figures (figures 10-27, 10-28) that include scanning electron micrographs of cells before and after they begin apoptosis and diagrams outlining the pathways involved in apoptosis. Other examples include new information that has been added to chapter 17 to discuss the process of licensing eukaryotic DNA for replication and the role of ATM and $\mathrm{Mdm} 2$ in regulating $\mathrm{p} 53$.

The clear illustrations in TWC, many of which have been updated since the fourth edition, are a major strength of the book. Many illustrations show processes in a step-by-step fashion and have captions placed within the figures that outline the steps of the process (e.g., figure 8-12, which illustrates the mechanism of the $\mathrm{Na}^{+} / \mathrm{K}^{+}$pump, and figure 20-16, which shows a model for the cotranslational import of polypeptides into the endoplasmic reticulum). Other figures show the relationships between different cellular processes, helping to integrate material. In this regard, one of my favorite figures on the topic of chemotrophic energy metabolism is figure 14-1, which nicely shows the relationship between glycolysis in the cytosol and the TCA cycle and electron transport chain within mitochondria. Most of the figures from the text are available in JPEG or Microsoft PowerPoint format on an instructor's CD-ROM. Although many excellent micrographs are presented throughout the book, these are not included in the instructor's CD-ROM.

In addition to the instructor's CD-ROM with illustrations, the fifth edition of TWC has several other supplemental materials available, including a booklet on microscopy packaged with the text, a set of transparencies, computerized and printed test banks, and a series of online labs. New with the fifth edition is a CD-ROM for students that is packaged with the book and is available with or without solutions to the problem sets at the end of chapters. The student CD has errors, such as incomplete questions in the quizzes and a mix-up of the quizzes for chapters 9 and 10, that I hope will be corrected in the next edition. In the sample student CDROM that I obtained, I was not able to create a deck of flashcards with terms from a single chapter, even though the Help manual provided instructions for doing so. Another new supplement to the fifth edition is The Benjamin Cummings Special Topics In Biology Series, a selection of four short booklets on cancer, stem cells, biological terrorism, and the human genome project. The stem cell booklet has been reviewed recently (Fink, 2004). I have primarily examined and used information from the booklet titled Biology of Cancer (Phillis and Goodwin, 2003). This booklet is written at a level suitable for general biology students but also could be used by advanced students to complement more detailed reading on cell cycle regulation and cancer.

The fifth edition of TWC is a textbook of manageable size that provides an up-to-date coverage of cell biology. The text is succinct and well written. For an undergraduate cell biology course, I like the author's approach of presenting key principles of cell biology "without obscuring the forest for the trees." If you would like to explore TWC further, I recommend examining the sample chapters available online at the publisher's Web site (http://www.aw-bc.com/info/ becker/).

\section{ACKNOWLEDGMENTS}

I thank Lisa Montplaisir and Robin Wright for comments and suggestions on the manuscript.

\section{REFERENCES}

Fink, R. (2004). A shelf of stem cells. Cell Biol. Educ. 3, 20-21.

Phillis, R.W., and Goodwin, S. (2003). Biology of Cancer. San Francisco, CA: Benjamin Cummings. 